\title{
Additive Refinements and Reverses of Young's Operator Inequality Via a Result of Cartwright and Field
}

\author{
Silvestru Sever Dragomir ${ }^{1,2^{*}}$ \\ ${ }^{1}$ Mathematics, College of Engineering \& Science, Victoria University, PO Box 14428, Melbourne City, MC 8001, Australia \\ ${ }^{2}$ School of Computer Science \& Applied Mathematics, University of the Witwatersrand, Private Bag 3, Johannesburg 2050, South Africa
}

\section{Article Info}

Keywords: Arithmetic mean-Geometric mean inequality, Hölder-McCarthy operator inequality, Young's inequality. 2010 AMS: 47A63, 47A30, 15A60, 26D15; $26 D 10$

Received: 30 November 2020

Accepted: 1 March 2021

Available online: 22 March 2021

\begin{abstract}
In this paper we obtain some new additive refinements and reverses of Young's operator inequality via a result of Cartwright and Field. Comparison with other additive Young's type inequalities are also provided.
\end{abstract}

\section{Introduction}

We have the following inequality that provides a refinement and a reverse for the celebrated Young's inequality

$$
\frac{1}{2} v(1-v) \frac{(b-a)^{2}}{\max \{a, b\}} \leq(1-v) a+v b-a^{1-v} b^{v} \leq \frac{1}{2} v(1-v) \frac{(b-a)^{2}}{\min \{a, b\}}
$$

for any $a, b>0$ and $v \in[0,1]$.

This result was obtained in 1978 by Cartwright and Field [1] who established a more general result for $n$ variables and gave an application for a probability measure supported on a finite interval.

Throughout this paper $A, B$ are positive invertible operators on a complex Hilbert space $(H,\langle\cdot, \cdot\rangle)$. We use the following notations for operators

$$
A \nabla_{v} B:=(1-v) A+v B,
$$

the weighted operator arithmetic mean and

$$
A \sharp_{v} B:=A^{1 / 2}\left(A^{-1 / 2} B A^{-1 / 2}\right)^{v} A^{1 / 2},
$$

the weighted operator geometric mean. When $v=\frac{1}{2}$ we write $A \nabla B$ and $A \sharp B$ for brevity, respectively.

The famous Young inequality for positive invertible operators $A, B$ says that if $v \in[0,1]$, then

$$
A \sharp_{v} B \leq A \nabla_{v} B .
$$

The inequality (1.2) is also called $v$-weighted arithmetic-geometric operator mean inequality.

In the recent paper [12], by the use of Cartwright and Field inequality (1.1), Minculete and Furuichi showed amongst other that

$$
\begin{aligned}
\frac{1}{2} v(1-v)\left(A B^{-1} A-2 A+B\right) & \leq A \nabla_{v} B-A \sharp_{v} B \\
& \leq \frac{1}{2} v(1-v)\left(B A^{-1} B-2 B+A\right),
\end{aligned}
$$


provided that $A \leq B$, and

$$
\begin{aligned}
\frac{1}{2} v(1-v)\left(B A^{-1} B-2 B+A\right) & \leq A \nabla_{v} B-A \sharp_{v} B \\
& \leq \frac{1}{2} v(1-v)\left(A B^{-1} A-2 A+B\right),
\end{aligned}
$$

provided that $B \leq A$.

For other inequalities between the operator means $A \sharp_{v} B$ and $A \nabla_{v} B$ see [2]- [11], [13]- [14] and the references therein.

In this paper, several other lower and upper bounds for the Young's difference $A \nabla_{v} B-A \sharp_{v} B$ under various boundedness assumptions for the involved operators $A$ and $B$ are given. Comparison with other additive Young's type inequalities are also provided.

\section{A Refinement and Reverse of Young's Inequality}

We have:

Theorem 2.1. Let $A, B$ be positive invertible operators and $M>m>0$ such that

$$
M A \geq B \geq m A .
$$

Then for any $v \in[0,1]$ we have

$$
\begin{aligned}
\frac{1}{2} v(1-v) c(m, M) A & \leq \frac{1}{2} \frac{v(1-v)}{\max \{M, 1\}}(B-A) A^{-1}(B-A), \\
& \leq A \nabla_{v} B-A \sharp_{v} B, \\
& \leq \frac{1}{2} \frac{v(1-v)}{\min \{m, 1\}},(B-A) A^{-1}(B-A), \\
& \leq \frac{1}{2} v(1-v) C(m, M) A,
\end{aligned}
$$

where

$$
c(m, M):=\left\{\begin{array}{l}
(M-1)^{2} \text { if } M<1 \\
0 \text { if } m \leq 1 \leq M \\
\frac{(m-1)^{2}}{M} \text { if } 1<m
\end{array}\right.
$$

and

$$
C(m, M):=\left\{\begin{array}{l}
\frac{(m-1)^{2}}{m} \text { if } M<1, \\
\frac{1}{m} \max \left\{(m-1)^{2},(M-1)^{2}\right\} \text { if } m \leq 1 \leq M \\
(M-1)^{2} \text { if } 1<m .
\end{array}\right.
$$

In particular,

$$
\begin{aligned}
\frac{1}{8} c(m, M) A & \leq \frac{1}{8 \max \{M, 1\}}(B-A) A^{-1}(B-A) \leq A \nabla B-A \sharp B \\
& \leq \frac{1}{8 \min \{m, 1\}}(B-A) A^{-1}(B-A) \leq \frac{1}{8} C(m, M) A .
\end{aligned}
$$

Proof. If we write the inequality (1.1) for $a=1$ and $b=x$ we get

$$
\frac{1}{2} v(1-v) \frac{(x-1)^{2}}{\max \{x, 1\}} \leq 1-v+v x-x^{v} \leq \frac{1}{2} v(1-v) \frac{(x-1)^{2}}{\min \{x, 1\}}
$$

for any $x>0$ and for any $v \in[0,1]$.

If $x \in[m, M] \subset(0, \infty)$, then $\max \{x, 1\} \leq \max \{M, 1\}$ and $\min \{m, 1\} \leq \min \{x, 1\}$ and by (2.4) we get

$$
\begin{aligned}
\frac{1}{2} v(1-v) \frac{\min _{x \in[m, M]}(x-1)^{2}}{\max \{M, 1\}} & \leq \frac{1}{2} v(1-v) \frac{(x-1)^{2}}{\max \{M, 1\}} \\
& \leq 1-v+v x-x^{v} \\
& \leq \frac{1}{2} v(1-v) \frac{(x-1)^{2}}{\min \{m, 1\}} \\
& \leq \frac{1}{2} v(1-v) \frac{\max _{x \in[m, M]}(x-1)^{2}}{\min \{m, 1\}}
\end{aligned}
$$

for any $x \in[m, M]$ and for any $v \in[0,1]$. 
Observe that

$$
\min _{x \in[m, M]}(x-1)^{2}=\left\{\begin{array}{l}
(M-1)^{2} \text { if } M<1, \\
0 \text { if } m \leq 1 \leq M, \\
(m-1)^{2} \text { if } 1<m
\end{array}\right.
$$

and

$$
\max _{x \in[m, M]}(x-1)^{2}=\left\{\begin{array}{l}
(m-1)^{2} \text { if } M<1, \\
\max \left\{(m-1)^{2},(M-1)^{2}\right\} \text { if } m \leq 1 \leq M, \\
(M-1)^{2} \text { if } 1<m .
\end{array}\right.
$$

Then

$$
\frac{\min _{x \in[m, M]}(x-1)^{2}}{\max \{M, 1\}}=\left\{\begin{array}{l}
(M-1)^{2} \text { if } M<1, \\
0 \text { if } m \leq 1 \leq M, \quad=c(m, M) \\
\frac{(m-1)^{2}}{M} \text { if } 1<m
\end{array}\right.
$$

and

$$
\frac{\max _{x \in[m, M]}(x-1)^{2}}{\min \{m, 1\}}=\left\{\begin{array}{l}
\frac{(m-1)^{2}}{m} \text { if } M<1, \\
\frac{1}{m} \max \left\{(m-1)^{2},(M-1)^{2}\right\} \text { if } m \leq 1 \leq M, \quad=C(m, M) \\
(M-1)^{2} \text { if } 1<m .
\end{array}\right.
$$

Using the inequality (2.5) we have

$$
\begin{aligned}
\frac{1}{2} v(1-v) c(m, M) & \leq \frac{1}{2} v(1-v) \frac{(x-1)^{2}}{\max \{M, 1\}} \\
& \leq 1-v+v x-x^{v} \\
& \leq \frac{1}{2} v(1-v) \frac{(x-1)^{2}}{\min \{m, 1\}} \\
& \leq \frac{1}{2} v(1-v) C(m, M)
\end{aligned}
$$

for any $x \in[m, M]$ and for any $v \in[0,1]$.

If we use the continuous functional calculus for the positive invertible operator $X$ with $m I \leq X \leq M I$, then we have from (2.10) that

$$
\begin{aligned}
\frac{1}{2} v(1-v) c(m, M) I & \leq \frac{1}{2} \frac{v(1-v)}{\max \{M, 1\}}(X-I)^{2} \\
& \leq(1-v) I+v X-X^{v} \\
& \leq \frac{1}{2} \frac{v(1-v)}{\min \{m, 1\}}(X-I)^{2} \\
& \leq \frac{1}{2} v(1-v) C(m, M) I
\end{aligned}
$$

for any $v \in[0,1]$.

If we multiply (2.1) both sides by $A^{-1 / 2}$ we get $M I \geq A^{-1 / 2} B A^{-1 / 2} \geq m I$.

By writing the inequality (2.7) for $X=A^{-1 / 2} B A^{-1 / 2}$ we obtain

$$
\begin{aligned}
\frac{1}{2} v(1-v) c(m, M) I & \leq \frac{1}{2} \frac{v(1-v)}{\max \{M, 1\}}\left(A^{-1 / 2} B A^{-1 / 2}-I\right)^{2} \\
& \leq(1-v) I+v A^{-1 / 2} B A^{-1 / 2}-\left(A^{-1 / 2} B A^{-1 / 2}\right)^{v} \\
& \leq \frac{1}{2} \frac{v(1-v)}{\min \{m, 1\}}\left(A^{-1 / 2} B A^{-1 / 2}-I\right)^{2} \\
& \leq \frac{1}{2} v(1-v) C(m, M) I
\end{aligned}
$$

for any $v \in[0,1]$. 
If we multiply the inequality (2.8) both sides with $A^{1 / 2}$, then we get

$$
\begin{aligned}
\frac{1}{2} v(1-v) c(m, M) A & \leq \frac{1}{2} \frac{v(1-v)}{\max \{M, 1\}} A^{1 / 2}\left(A^{-1 / 2} B A^{-1 / 2}-I\right)^{2} A^{1 / 2} \\
& \leq(1-v) A+v B-A^{1 / 2}\left(A^{-1 / 2} B A^{-1 / 2}\right)^{v} A^{1 / 2} \\
& \leq \frac{1}{2} \frac{v(1-v)}{\min \{m, 1\}} A^{1 / 2}\left(A^{-1 / 2} B A^{-1 / 2}-I\right)^{2} A^{1 / 2} \\
& \leq \frac{1}{2} v(1-v) C(m, M) A,
\end{aligned}
$$

and since

$$
\begin{aligned}
A^{1 / 2}\left(A^{-1 / 2} B A^{-1 / 2}-I\right)^{2} A^{1 / 2} & =A^{1 / 2}\left(A^{-1 / 2}(B-A) A^{-1 / 2}\right)^{2} A^{1 / 2} \\
& =A^{1 / 2} A^{-1 / 2}(B-A) A^{-1 / 2} A^{-1 / 2}(B-A) A^{-1 / 2} A^{1 / 2} \\
& =(B-A) A^{-1}(B-A),
\end{aligned}
$$

then by (2.9) we get the desired result (2.2).

When the operators $A$ and $B$ are bounded above and below by constants we have the following result as well:

Corollary 2.2. Let $A, B$ be two positive operators and $m, m^{\prime}, M, M^{\prime}$ be positive real numbers. Put $h:=\frac{M}{m}$ and $h^{\prime}:=\frac{M^{\prime}}{m^{\prime}}$. (i) If $0<m I \leq A \leq m^{\prime} I<M^{\prime} I \leq B \leq M I$, then

$$
\begin{aligned}
\frac{1}{2} v(1-v) \frac{\left(h^{\prime}-1\right)^{2}}{h} A & \leq \frac{1}{2} \frac{v(1-v)}{h}(B-A) A^{-1}(B-A) \\
& \leq A \nabla_{v} B-A \sharp_{v} B \\
& \leq \frac{1}{2} v(1-v)(B-A) A^{-1}(B-A) \\
& \leq \frac{1}{2} v(1-v)(h-1)^{2} A,
\end{aligned}
$$

and, in particular,

$$
\begin{aligned}
\frac{\left(h^{\prime}-1\right)^{2}}{8 h} A & \leq \frac{1}{8 h}(B-A) A^{-1}(B-A) \leq A \nabla B-A \sharp B \\
& \leq \frac{1}{8}(B-A) A^{-1}(B-A) \leq \frac{1}{8}(h-1)^{2} A .
\end{aligned}
$$

(ii) If $0<m I \leq B \leq m^{\prime} I<M^{\prime} I \leq A \leq M I$, then

$$
\begin{aligned}
\frac{1}{2} v(1-v)\left(\frac{h^{\prime}-1}{h^{\prime}}\right)^{2} A & \leq \frac{1}{2} v(1-v)(B-A) A^{-1}(B-A) \\
& \leq A \nabla_{v} B-A \sharp_{v} B \\
& \leq \frac{1}{2} v(1-v) h(B-A) A^{-1}(B-A) \\
& \leq \frac{1}{2} v(1-v) \frac{(h-1)^{2}}{h} A
\end{aligned}
$$

and, in particular,

$$
\begin{aligned}
\frac{1}{8}\left(\frac{h^{\prime}-1}{h^{\prime}}\right)^{2} A & \leq \frac{1}{8}(B-A) A^{-1}(B-A) \leq A \nabla B-A \sharp B \\
& \leq \frac{1}{8} h(B-A) A^{-1}(B-A) \leq \frac{(h-1)^{2}}{8 h} A .
\end{aligned}
$$

Proof. We observe that $h, h^{\prime}>1$ and if either of the condition (i) or (ii) holds, then $h \geq h^{\prime}$. If (i) is valid, then we have

$$
A<h^{\prime} A=\frac{M^{\prime}}{m^{\prime}} A \leq B \leq \frac{M}{m} A=h A,
$$

while, if (ii) is valid, then we have

$$
\frac{1}{h} A \leq B \leq \frac{1}{h^{\prime}} A<A .
$$

If we use the inequality (2.2) and the assumption (i), then we get (2.10).

If we use the inequality (2.2) and the assumption (ii), then we get (2.12). 


\section{Bounds in Term of Kantorovich's Constant}

We consider the Kantorovich's constant defined by

$$
K(h):=\frac{(h+1)^{2}}{4 h}, h>0 .
$$

The function $K$ is decreasing on $(0,1)$ and increasing on $[1, \infty), K(h) \geq 1$ for any $h>0$ and $K(h)=K\left(\frac{1}{h}\right)$ for any $h>0$. Observe that for any $h>0$

$$
K(h)-1=\frac{(h-1)^{2}}{4 h}=K\left(\frac{1}{h}\right)-1 .
$$

Also, if $a, b>0$ then

$$
K\left(\frac{b}{a}\right)-1=\frac{(b-a)^{2}}{4 a b} .
$$

Since $\min \{a, b\} \max \{a, b\}=a b$ if $a, b>0$, then

$$
\frac{(b-a)^{2}}{\max \{a, b\}}=\frac{\min \{a, b\}(b-a)^{2}}{a b}=4 \min \{a, b\}\left[K\left(\frac{b}{a}\right)-1\right]
$$

and

$$
\frac{(b-a)^{2}}{\min \{a, b\}}=\frac{\max \{a, b\}(b-a)^{2}}{a b}=4 \max \{a, b\}\left[K\left(\frac{b}{a}\right)-1\right]
$$

and the inequality (1.1) can be written as

$$
\begin{aligned}
2 v(1-v) \min \{a, b\}\left[K\left(\frac{b}{a}\right)-1\right] & \leq(1-v) a+v b-a^{1-v} b^{v} \\
& \leq 2 v(1-v) \max \{a, b\}\left[K\left(\frac{b}{a}\right)-1\right]
\end{aligned}
$$

for any $a, b>0$ and $v \in[0,1]$.

For positive invertible operators $A, B$ we define

$$
A \nabla_{\infty} B:=\frac{1}{2}(A+B)+\frac{1}{2} A^{1 / 2}\left|A^{-1 / 2}(B-A) A^{-1 / 2}\right| A^{1 / 2}
$$

and

$$
A \nabla_{-\infty} B:=\frac{1}{2}(A+B)-\frac{1}{2} A^{1 / 2}\left|A^{-1 / 2}(B-A) A^{-1 / 2}\right| A^{1 / 2} .
$$

If we consider the continuous functions $f_{\infty}, f_{-\infty}:[0, \infty) \rightarrow[0, \infty)$ defined by

$$
f_{\infty}(x)=\max \{x, 1\}=\frac{1}{2}(x+1)+\frac{1}{2}|x-1|
$$

and

$$
f_{-\infty}(x)=\max \{x, 1\}=\frac{1}{2}(x+1)-\frac{1}{2}|x-1|,
$$

then, obviously, we have

$$
A \nabla_{ \pm \infty} B=A^{1 / 2} f_{ \pm \infty}\left(A^{-1 / 2} B A^{-1}\right) A^{1 / 2} .
$$

If $A$ and $B$ are commutative, then

$$
A \nabla_{ \pm \infty} B=\frac{1}{2}(A+B) \pm \frac{1}{2}|B-A|=B \nabla_{ \pm \infty} A .
$$

Theorem 3.1. Let $A, B$ be positive invertible operators and $M>m>0$ such that the condition (2.1) holds. Then we have

$$
\begin{aligned}
2 v(1-v) g(m, M) A \nabla_{-\infty} B & \leq A \nabla_{v} B-A \sharp_{v} B \\
& \leq 2 v(1-v) G(m, M) A \nabla_{\infty} B,
\end{aligned}
$$

where

$$
g(m, M):=\left\{\begin{array}{l}
K(M)-1 \text { if } M<1, \\
0 \text { if } m \leq 1 \leq M \\
K(m)-1 \text { if } 1<m
\end{array}\right.
$$


and

$$
G(m, M):=\left\{\begin{array}{l}
K(m)-1 \text { if } M<1, \\
\max \{K(m), K(M)\}-1 \text { if } m \leq 1 \leq M, \\
K(M)-1 \text { if } 1<m .
\end{array}\right.
$$

In particular,

$$
\frac{1}{2} g(m, M) A \nabla_{-\infty} B \leq A \nabla B-A \sharp B \leq \frac{1}{2} G(m, M) A \nabla_{\infty} B .
$$

Proof. From (3.2) we have for $a=1$ and $b=x$ that

$$
\begin{aligned}
2 v(1-v) \min \{1, x\}[K(x)-1] & \leq 1-v+v x-x^{v} \\
& \leq 2 v(1-v) \max \{1, x\}[K(x)-1]
\end{aligned}
$$

for any $x>0$.

From (3.6) we then have

$$
\begin{aligned}
2 v(1-v) f_{-\infty}(x) \min _{x \in[m, M]}[K(x)-1] & \leq 1-v+v x-x^{v} \\
& \leq 2 v(1-v) f_{\infty}(x) \max _{x \in[m, M]}[K(x)-1]
\end{aligned}
$$

for any $x \in[m, M]$.

Observe that

$$
\max _{x \in[m, M]}[K(x)-1]=\left\{\begin{array}{l}
K(m)-1 \text { if } M<1, \\
\max \{K(m), K(M)\}-1 \text { if } m \leq 1 \leq M, \quad=G(m, M) \\
K(M)-1 \text { if } 1<m .
\end{array}\right.
$$

and

$$
\min _{x \in[m, M]}[K(x)-1]=\left\{\begin{array}{l}
K(M)-1 \text { if } M<1, \\
0 \text { if } m \leq 1 \leq M, \\
K(m)-1 \text { if } 1<m .
\end{array}=g(m, M) .\right.
$$

Therefore by (3.7) we get

$$
2 v(1-v) f_{-\infty}(x) g(m, M) \leq 1-v+v x-x^{v} \leq 2 v(1-v) f_{\infty}(x) G(m, M)
$$

for any $x \in[m, M]$ and $v \in[0,1]$.

If we use the continuous functional calculus for the positive invertible operator $X$ with $m I \leq X \leq M I$, then we have from (3.8) that

$$
\begin{aligned}
2 v(1-v) f_{-\infty}(X) g(m, M) & \leq(1-v) I+v X-X^{v} \\
& \leq 2 v(1-v) f_{\infty}(X) G(m, M)
\end{aligned}
$$

for any $x \in[m, M]$ and $v \in[0,1]$.

By writing the inequality (2.7) for $X=A^{-1 / 2} B A^{-1 / 2}$ we obtain

$$
\begin{aligned}
2 v(1-v) f_{-\infty}\left(A^{-1 / 2} B A^{-1 / 2}\right) g(m, M) & \leq(1-v) I+v A^{-1 / 2} B A^{-1 / 2}-\left(A^{-1 / 2} B A^{-1 / 2}\right)^{v} \\
& \leq 2 v(1-v) f_{\infty}\left(A^{-1 / 2} B A^{-1 / 2}\right) G(m, M)
\end{aligned}
$$

for any $v \in[0,1]$

If we multiply (3.10) both sides by $A^{1 / 2}$ we get

$$
\begin{aligned}
2 v(1-v) A^{1 / 2} f_{-\infty}\left(A^{-1 / 2} B A^{-1 / 2}\right) A^{1 / 2} g(m, M) & \leq(1-v) A+v B A-A^{1 / 2}\left(A^{-1 / 2} B A^{-1 / 2}\right)^{v} A^{1 / 2} \\
& \leq 2 v(1-v) A^{1 / 2} f_{\infty}\left(A^{-1 / 2} B A^{-1 / 2}\right) A^{1 / 2} G(m, M)
\end{aligned}
$$

for any $v \in[0,1]$, which, by (3.3) produces the desired result (3.4).

We have: 
Corollary 3.2. Let $A, B$ be two positive operators and $m, m^{\prime}, M, M^{\prime}$ be positive real numbers. Put $h:=\frac{M}{m}$ and $h^{\prime}:=\frac{M^{\prime}}{m^{\prime}}$. If either of the conditions (i) or (ii) from Corollary 2.2 holds, then

$$
\begin{aligned}
2 v(1-v)\left[K\left(h^{\prime}\right)-1\right] A \nabla_{-\infty} B & \leq A \nabla_{v} B-A \sharp_{v} B \\
& \leq 2 v(1-v)[K(h)-1] A \nabla_{\infty} B .
\end{aligned}
$$

In particular,

$$
\frac{1}{2}\left[K\left(h^{\prime}\right)-1\right] A \nabla_{-\infty} B \leq A \nabla B-A \sharp B \leq \frac{1}{2}[K(h)-1] A \nabla_{\infty} B .
$$

Proof. If (i) is valid, then we have

$$
A<h^{\prime} A=\frac{M^{\prime}}{m^{\prime}} A \leq B \leq \frac{M}{m} A=h A .
$$

By using the inequality (3.4) we get (3.11).

If (ii) is valid, then we have

$$
\frac{1}{h} A \leq B \leq \frac{1}{h^{\prime}} A<A .
$$

By using the inequality (3.4) we get

$$
\begin{aligned}
2 v(1-v)\left[K\left(\frac{1}{h^{\prime}}\right)-1\right] A \nabla_{-\infty} B & \leq A \nabla_{v} B-A \sharp_{v} B \\
& \leq 2 v(1-v)\left[K\left(\frac{1}{h}\right)-1\right] A \nabla_{\infty} B,
\end{aligned}
$$

and since $K\left(\frac{1}{h^{\prime}}\right)=K\left(h^{\prime}\right)$ and $K\left(\frac{1}{h}\right)=K(h)$, the inequality (3.11) is also obtained.

\section{Comparison with Other Additive Inequalities}

Kittaneh and Manasrah [9], [10] provided a refinement and a reverse for Young's scalar inequality as follows:

$$
r(\sqrt{a}-\sqrt{b})^{2} \leq(1-v) a+v b-a^{1-v} b^{v} \leq R(\sqrt{a}-\sqrt{b})^{2}
$$

where $a, b>0, v \in[0,1], r=\min \{1-v, v\}$ and $R=\max \{1-v, v\}$. The case $v=\frac{1}{2}$ reduces (4.1) to an identity and is of no interest. In [2] we obtained the following logarithmic upper bound for the Young's difference

$$
(0 \leq)(1-v) a+v b-a^{1-v} b^{v} \leq v(1-v)(a-b)(\ln a-\ln b)
$$

for any $a, b>0$ and $v \in[0,1]$, while in the subsequent paper [3] we obtained the following refinement and reverse of Young's inequality

$$
\begin{aligned}
\frac{1}{2} v(1-v)(\ln a-\ln b)^{2} \min \{a, b\} & \leq(1-v) a+v b-a^{1-v} b^{v} \\
& \leq \frac{1}{2} v(1-v)(\ln a-\ln b)^{2} \max \{a, b\},
\end{aligned}
$$

for any $a, b>0$ and $v \in[0,1]$.

Consider the following functions of two variables obtained from the upper bounds in inequalities (1.1), (4.1), (4.2) and (4.3) for $a=1$, $b=x \in(0, \infty)$ and $v=y \in(0,1)$, namely

$$
\begin{aligned}
& U_{1}(x, y):=\frac{1}{2} y(1-y) \frac{(x-1)^{2}}{\min \{x, 1\}}, \\
& U_{2}(x, y):=\max \{y, 1-y\}(\sqrt{x}-1)^{2}, \\
& U_{3}(x, y):=y(1-y)(x-1) \ln x \text { and } \\
& U_{4}(x, y):=\frac{1}{2} y(1-y) \max \{x, 1\} \ln ^{2} x .
\end{aligned}
$$

We observe that the $3 \mathrm{D}$ plots of the differences $U_{1}(x, y)-U_{2}(x, y)$ on $(0,10) \times(0,1), U_{1}(x, y)-U_{3}(x, y)$ on $(2,4) \times(0,1), U_{2}(x, y)-U_{3}(x, y)$ on $(2,4) \times(0,1), U_{2}(x, y)-U_{4}(x, y)$ on $(2,4) \times(0,1)$ and $U_{3}(x, y)-U_{4}(x, y)$ on $(3,6) \times(0,1)$ show that they take both negative and positive values, meaning that neither of the corresponding upper bounds are better in general.

It appears that $U_{1}(x, y)>U_{4}(x, y)$ on the box $(0,10) \times(0,1)$ suggesting that the upper bound in (4.3) is better than the one from (1.1). However we do not have an analytic proof for it in general.

Similar conclusions may be derived for the lower bounds, however the details are left to the interested reader. 


\section{Conclusion}

In this paper we obtained some new additive refinements and reverses of Young's operator inequality via a result of Cartwright and Field. Comparison with other additive Young's type inequalities were also provided.

\section{References}

[1] D.I. Cartwright, M.J. Field, A refinement of the arithmetic mean-geometric mean inequality, Proc. Amer. Math. Soc., 71 (1978), $36-38$.

[2] S.S. Dragomir, Some new reverses of Young's operator inequality, Preprint RGMIA Res. Rep. Coll. 18 (2015), Art. 130. [http://rgmia.org/papers/v18/v18a130.pdf].

[3] S.S. Dragomir, On new refinements and reverses of Young's operator inequality, Preprint RGMIA Res. Rep. Coll. 18 (2015), Art. 135. [http://rgmia.org/papers/v18/v18a135.pdf].

[4] S.S. Dragomir, Some inequalities for operator weighted geometric mean, Preprint RGMIA Res. Rep. Coll. 18 (2015), Art. 139. [http://rgmia.org/papers/v18/v18a139.pdf ].

[5] S.S. Dragomir, Refinements and reverses of Hölder-McCarthy operator inequality, Preprint RGMIA Res. Rep. Coll. 18 (2015), Art. 143. [http://rgmia.org/papers/v18/v18a143.pdf]

[6] S.S. Dragomir, Some reverses and a refinement of Hölder operator inequality, Preprint RGMIA Res. Rep. Coll. 18 (2015), Art. 147. [http://rgmia.org/papers/v18/v18a147.pdf].

[7] S. Furuichi, Refined Young inequalities with Specht's ratio, J. Egyptian Math. Soc. 20(2012), 46-49.

[8] S. Furuichi, On refined Young inequalities and reverse inequalities, J. Math. Inequal. 5 (2011), 21-31.

[9] F. Kittaneh, Y. Manasrah, Improved Young and Heinz inequalities for matrix, J. Math. Anal. Appl., 361 (2010), $262-269$

[10] F. Kittaneh, Y. Manasrah, Reverse Young and Heinz inequalities for matrices, Linear Multilinear Algebra., 59 (2011), $1031-1037$.

[11] W. Liao, J. Wu, J. Zhao, New versions of reverse Young and Heinz mean inequalities with the Kantorovich constant, Taiwanese J. Math. 19(2) (2015), $467-479$.

[12] N. Minculete, S. Furuichi, Several applications of Cartwright-Field's inequality, Int. J. Pure Appl. Math., 71(1) (21), 19-30.

[13] M. Tominaga, Specht's ratio in the Young inequality, Sci. Math. Japon., 55 (2002), 583-588.

[14] G. Zuo, G. Shi, M. Fujii, Refined Young inequality with Kantorovich constant, J. Math. Inequal., 5 (2011), 551-556. 